FACTA UNIVERSITATIS (NIŠ)

Ser. Math. Inform. Vol. 33, No 3 (2018), 361-373

https://doi.org/10.22190/FUMI1803361P

\title{
ON m-PROJECTIVE CURVATURE TENSOR OF GENERALIZED SASAKIAN-SPACE-FORMS
}

\author{
Shravan K. Pandey and R.N. Singh
}

\begin{abstract}
The aim of the paper is to characterize generalized Sasakian-space-forms satisfying certain curvature conditions on the m-projective curvature tensor. We study m-projectively semisymmetric, m-projectively flat, $\xi$-m-projectively flat, and m-proje-ctively recurrent generalized Sasakian-space-forms. $W^{*} . S=0$ and $W^{*} \cdot R=0$ on generalized Sasakian-space-forms are also studied.

Keywords: generalized Sasakian-space-forms, m-projectively semisymmetric, $\mathrm{m}$-projectively flat, m-projectively recurrent, $\xi$-m-projectively flat.
\end{abstract}

\section{Introduction}

Studying the almost Hermitian manifold, Alfred Gray, a well-known geometrician, formulated a principle according to which the so-called curvature identities for the Riemann-Christoffel tensor are key to understanding differential-geometric properties of such manifolds 13 . Many papers are devoted to the study of geometric consequences of these identities and to their analogs for almost contact metric structures. As a continuation of this line of research, we consider some curvature properties of generalized Sasakian-space-forms regarding the m-projective curvature tensor.

A generalized Sasakian-space-form was defined by P. Alegre, D. E. Blair and A. Carriazo in [1] as an almost contact metric manifold $\left(M^{2 n+1}, \phi, \xi, \eta, \mathrm{g}\right)$ whose curvature tensor $\mathrm{R}$ is given by

$$
R=f_{1} R_{1}+f_{2} R_{2}+f_{3} R_{3},
$$

where $f_{1}, f_{2}, f_{3}$ are some differential functions on $M^{2 n+1}$ and

$$
\begin{gathered}
R_{1}(X, Y) Z=g(Y, Z) X-g(X, Z) Y, \\
R_{2}(X, Y) Z=g(X, \phi Z) \phi Y-g(Y, \phi Z) \phi X+2 g(X, \phi Y) \phi Z,
\end{gathered}
$$

Received December 05, 2016; accepted June 10, 2017.

2010 Mathematics Subject Classification. Primary 53C25; Secondary 53D15 


$$
R_{3}(X, Y) Z=\eta(X) \eta(Z) Y-\eta(Y) \eta(Z) X+g(X, Z) \eta(Y) \xi-g(Y, Z) \eta(X) \xi,
$$

for any vector field $\mathrm{X}, \mathrm{Y}, \mathrm{Z}$ on $M^{2 n+1}$. In such a case we denote the manifold as $M\left(f_{1}, f_{2}, f_{3}\right)$. This kind of manifold appears as a generalization of the wellknown Sasakian-space-forms by taking $f_{1}=\frac{c+3}{4}, f_{2}=f_{3}=\frac{c-1}{4}$. It is known that any three-dimensional $(\alpha, \beta)$-trans-Sasakian manifold with $\alpha, \beta$ depending on $\xi$ is a generalized Sasakian-space-form [2].P.Alegre, A.Carriazo, Y.H.Kim and D.W.Yoon give results in [3] about B.Y.Chen's inequality on submanifolds of generalized complex space-forms and generalized Sasakian-space-forms. R. Al-Ghefari, F.R. Al-Solamy and M.H.Shahid analyse in [4] and [5] CR-submanifolds of generalized Sasakian-space-forms. In 9, U.K.Kim studied conformally flat generalized Sasakian-space-forms and locally symmetric generalized Sasakian-space-forms. U.C.De and A.Sarkar 7] studied generalized Sasakian-space-forms regarding the projective curvature tensor. On the other hand, in 1971, G.P.Pokhariyal and R.S.Mishra [12] defined a tensor field $W^{*}$ on a Riemannian manifold as

$$
\begin{aligned}
{ }^{\prime} W^{*}(X, Y, Z, U)= & { }^{\prime} R(X, Y, Z, U)-\frac{1}{2(n-1)}[S(Y, Z) g(X, U)-S(X, Z) g(Y, U) \\
& +g(Y, Z) S(X, U)-g(X, Z) S(Y, U)],
\end{aligned}
$$

where ${ }^{\prime} W^{*}(X, Y, Z, U)=g\left(W^{*}(X, Y) Z, U\right)$ and ' $R(X, Y, Z, U)=g(R(X, Y) Z, U)$. Such a tensor field $W^{*}$ is known as m-projective curvature tensor. Later, R. H.Ojha [10] defined and studied the properties of the m-projective curvature tensor in Sasakian and Kähler manifolds. He also showed that it bridges the gap between the conformal curvature tensor, conharmonic curvature tensor and concircular curvature tensor on one side and the $\mathrm{H}$-projective curvature tensor on the other.

Motivated by the above studies, we study here the flatness and symmetry property of generalized Sasakian-space-forms regarding the m-projective curvature tensor. The paper is organized as follows. In section 2, some preliminary results are recalled. In section 3, we study m-projectively semisymmetric generalized Sasakianspace-forms. Section 4 deals with m-projectively flat generalized Sasakian-spaceforms. $\xi$-m-projectively flat generalized Sasakian-space-forms are studied in Section 5 and necessary and sufficient condition are obtained for a generalized Sasakianspace-form to be $\xi$-m-projectively flat. In Section 6, m-projectively recurrent generalized Sasakian-space-forms are studied. Section 7 is devoted to the study of generalized Sasakian-space-forms satisfying $W^{*} . S=0$. The last section discusses generalized Sasakian-space-forms satisfying $W^{*} \cdot R=0$.

\section{Preliminaries}

If on an odd dimensional differentiable manifold $M^{2 n+1}$ of the differentiability class $C^{r+1}$ there exists a vector-valued real linear function $\phi$, a 1-form $\eta$, the associated 
vector field $\xi$ and the Riemannian metric g satisfying

$$
\begin{gathered}
\phi^{2} X=-X+\eta(X) \xi, \phi(\xi)=0, \\
\eta(\xi)=1, g(X, \xi)=\eta(X), \eta(\phi X)=0, \\
g(\phi X, \phi Y)=g(X, Y)-\eta(X) \eta(Y),
\end{gathered}
$$

for arbitrary vector fields $X$ and $Y$, then $\left(M^{2 n+1}, g\right)$ is said to be an almost contact metric manifold [6] and the structure $(\phi, \xi, \eta, g)$ is called an almost contact metric structure to $M^{2 n+1}$. In view of the equations (2.1), 2.2 and 2.3), we have

$$
\begin{gathered}
g(\phi X, Y)=-g(X, \phi Y), g(\phi X, X)=0, \\
\left(\nabla_{X} \eta\right)(Y)=g\left(\nabla_{X} \xi, Y\right) .
\end{gathered}
$$

Again we know that [1] in a $(2 n+1)$-dimensional generalized Sasakian-space-form

$$
\begin{aligned}
R(X, Y) Z & =f_{1}\{g(Y, Z) X-g(X, Z) Y\} \\
& +f_{2}\{g(X, \phi Z) \phi Y-g(Y, \phi Z) \phi X+2 g(X, \phi Y) \phi Z\} \\
& +f_{3}\{\eta(X) \eta(Z) Y-\eta(Y) \eta(Z) X+g(X, Z) \eta(Y) \xi-g(Y, Z) \eta(X) \xi\}
\end{aligned}
$$

for all vector fields $\mathrm{X}, \mathrm{Y}, \mathrm{Z}$ on $M^{2 n+1}$, where $\mathrm{R}$ denotes the curvature tensor of $M^{2 n+1}$.

$$
\begin{gathered}
S(X, Y)=\left(2 n f_{1}+3 f_{2}-f_{3}\right) g(X, Y)-\left(3 f_{2}+(2 n-1) f_{3}\right) \eta(X) \eta(Y), \\
Q X=\left(2 n f_{1}+3 f_{2}-f_{3}\right) X-\left(3 f_{2}+(2 n-1) f_{3}\right) \eta(X) \xi, \\
\left.r=2 n(2 n+1) f_{1}+6 n f_{2}-4 n f_{3}\right) .
\end{gathered}
$$

For generalized Sasakian-space-forms we also have

$$
\begin{gathered}
R(X, Y) \xi=\left(f_{1}-f_{3}\right)[\eta(Y) X-\eta(X) Y], \\
R(\xi, X) Y=-R(X, \xi) Y=\left(f_{1}-f_{3}\right)[g(X, Y) \xi-\eta(Y) X], \\
\eta(R(X, Y) Z)=\left(f_{1}-f_{3}\right)[\eta(X) g(Y, Z)-\eta(Y) g(X, Z)], \\
S(X, \xi)=2 n\left(f_{1}-f_{3}\right) \eta(X),
\end{gathered}
$$




$$
Q \xi=2 n\left(f_{1}-f_{3}\right) \xi
$$

where $Q$ is the Ricci operator, i.e. $g(Q X, Y)=S(X, Y)$.

A generalized Sasakian space-form is said to be $\eta$-Einstein if its Ricci tensor $S$ is of the form

$$
S(X, Y)=a g(X, Y)+b \eta(X) \eta(Y)
$$

for arbitrary vector fields $\mathrm{X}$ and $\mathrm{Y}$, where a and $\mathrm{b}$ are smooth functions on $M^{2 n+1}$. For a $(2 n+1)$-dimensional $(n>1)$ almost contact metric manifold the m-projective curvature tensor $W^{*}$ is given by 12 .

$W^{*}(X, Y) Z=R(X, Y) Z-\frac{1}{2(n-1)}[S(Y, Z) X-S(X, Z) Y+g(Y, Z) Q X-g(X, Z) Q Y]$.

The m-projective curvature tensor $W^{*}$ for a generalized Sasakian-space-form is given by

$$
W^{*}(X, Y) \xi=-\frac{\left(f_{1}-f_{3}\right)}{(n-1)}[\eta(Y) X-\eta(X) Y]-\frac{1}{2(n-1)}[\eta(Y) Q X-\eta(X) Q Y],
$$

$$
W^{*}(\xi, Y) Z=-\frac{\left(f_{1}-f_{3}\right)}{(n-1)}[g(Y, Z) \xi-\eta(Z) Y]-\frac{1}{2(n-1)}[S(Y, Z) \xi-\eta(Z) Q Y],
$$

$$
\begin{aligned}
\eta\left(W^{*}(\xi, Y) Z\right) & =-\frac{\left(f_{1}-f_{3}\right)}{(n-1)}[g(Y, Z)-\eta(Y) \eta(Z)] \\
& -\frac{1}{2(n-1)}\left[S(Y, Z)-2 n\left(f_{1}-f_{3}\right) \eta(Y) \eta(Z)\right]
\end{aligned}
$$

and

$$
\begin{aligned}
\eta\left(W^{*}(X, Y) Z\right) & =-\frac{\left(f_{1}-f_{3}\right)}{(n-1)}[g(Y, Z) \eta(X)-g(X, Z) \eta(Y)] \\
& -\frac{1}{2(n-1)}[S(Y, Z) \eta(X)-S(X, Z) \eta(Y)]
\end{aligned}
$$

\section{3. m-Projectively Semisymmetric Generalized Sasakian-Space-Forms}

Definition 3.1. A $(2 n+1)$-dimensional $(n>1)$ generalized Sasakian-space-form is said to be m-projectively semisymmetric [7] if it satisfies $\left(R(X, Y) \cdot W^{*}\right)(U, V) Z=$ 0 , where $R(X, Y)$ is to be considered a derivation of the tensor algebra at each point of the manifold for tangent vectors $X, Y$ and $W^{*}$ is the m-projective curvature tensor of the space-forms.

Theorem 3.1. If a $(2 n+1)$-dimensional $(n>1)$ generalized Sasakian-spaceform is m-projectively semisymmetric then either $f_{1}=f_{3}$ or $M^{2 n+1}$ is an Einstein manifold. 
Proof: Let us suppose that the generalized Sasakian-space-form is m-projectively semisymmetric. Then we can write

$$
\left(R(\xi, X) \cdot W^{*}\right)(Y, Z) U=0 .
$$

The above equation can be written as

$R(\xi, X) W^{*}(Y, Z) U-W^{*}(R(\xi, X) Y, Z) U-W^{*}(Y, R(\xi, X) Z) U-W^{*}(Y, Z) R(\xi, X) U=0$.

In view of the equation 2.11 the above equation reduces to

$$
\begin{aligned}
& \left(f_{1}-f_{3}\right)\left[g\left(X, W^{*}(Y, Z) U\right) \xi-\eta\left(W^{*}(Y, Z) U\right) X-g(X, Y) W^{*}(\xi, Z) U\right. \\
& +\eta(Y) W^{*}(X, Z) U-g(X, Z) W^{*}(Y, \xi) U+\eta(Z) W^{*}(Y, X) U \\
& \left.-g(X, U) W^{*}(Y, Z) \xi+\eta(U) W^{*}(Y, Z) X\right]=0 .
\end{aligned}
$$

Now, taking the inner product of the above equation with $\xi$ and using the equation (2.2), we get

$$
\begin{aligned}
& \left(f_{1}-f_{3}\right)\left[{ }^{\prime} W^{*}(Y, Z, U, X)-\eta\left(W^{*}(Y, Z) U\right) \eta(X)-g(X, Y) \eta\left(W^{*}(\xi, Z) U\right)\right. \\
& +\eta(Y) \eta\left(W^{*}(X, Z) U\right)-g(X, Z) \eta\left(W^{*}(Y, \xi) U\right)+\eta(Z) \eta\left(W^{*}(Y, X) U\right) \\
& \left.-g(X, U) \eta\left(W^{*}(Y, Z) \xi\right)+\eta(U) \eta\left(W^{*}(Y, Z) X\right)\right]=0,
\end{aligned}
$$

which on using the equations 2.18, 2.20) and (2.21) gives

$$
\begin{aligned}
& \left(f_{1}-f_{3}\right){ }^{\prime} R(Y, Z, U, X)-\frac{1}{2(n-1)}\{g(Z, U) S(Y, X)-g(Y, U) S(X, Z) \\
& +\{S(X, Z) \eta(Y)-S(X, Y) \eta(Z)\} \eta(U)+2 n\left(f_{1}-f_{3}\right)\{\eta(Z) g(X, Y) \\
& \left.-\eta(Y) g(X, Z)\} \eta(U)\}+\frac{\left(f_{1}-f_{3}\right)}{(n-1)}\{g(Z, U) g(X, Y)-g(Y, U) g(X, Z)\}\right] \\
& =0 .
\end{aligned}
$$

Putting $Z=U=e_{i}$ in the above equation and taking summation over i, $1 \leq i \leq$ $2 n+1$, we get

$$
\left(f_{1}-f_{3}\right)\left[S(X, Y)+(-n)\left(f_{1}-f_{3}\right) g(X, Y)\right]=0 .
$$

This gives either $f_{1}=f_{3}$ or

$$
S(X, Y)=n\left(f_{1}-f_{3}\right) g(X, Y),
$$

which shows that $M^{2 n+1}$ is an Einstein manifold. This completes the proof.

\section{4. m-Projectively Flat Generalized Sasakian-Space-Forms}

Theorem 4.1. $A(2 n+1)$-dimensional $(n>1)$ generalized Sasakian-space-form is m-projectively flat if and only if $f_{1}=\frac{3 f_{2}}{2(1-n)}=f_{3}$ provided any arbitrary vector field $Z$ is not pointwise collinear with the characteristic vector field $\xi$. 
Proof: For a $(2 n+1)$-dimensional $(n>1)$ m-projectively flat generalized Sasakianspace-form, we have from the equation 2.16

$$
R(X, Y) Z=\frac{1}{2(n-1)}[S(Y, Z) X-S(X, Z) Y+g(Y, Z) Q X-g(X, Z) Q Y]
$$

In view of the equations (2.7) and 2.8 the above equation takes the form

$$
\begin{aligned}
R(X, Y) Z & =\frac{1}{2(n-1)}\left[2\left(2 n f_{1}+3 f_{2}-f_{3}\right)\{g(Y, Z) X-g(X, Z) Y\}\right. \\
& -\left(3 f_{2}+(2 n-1) f_{3}\right)\{\eta(Y) X-\eta(X) Y\} \eta(Z) \\
& \left.-\left(3 f_{2}+(2 n-1) f_{3}\right)\{g(Y, Z) \eta(X)-g(X, Z) \eta(Y)\} \xi\right] .
\end{aligned}
$$

By virtue of the equation 2.6 the above equation reduces to

$$
\begin{aligned}
& f_{1}\{g(Y, Z) X-g(X, Z) Y\}+f_{2}\{g(X, \phi Z) \phi Y-g(Y, \phi Z) \phi X \\
& +2 g(X, \phi Y) \phi Z\}+f_{3}\{\eta(X) \eta(Z) Y-\eta(Y) \eta(Z) X+g(X, Z) \eta(Y) \xi \\
& -g(Y, Z) \eta(X) \xi\}=\frac{1}{2(n-1)}\left[2\left(2 n f_{1}+3 f_{2}-f_{3}\right)\{g(Y, Z) X-g(X, Z) Y\}\right. \\
& -\left(3 f_{2}+(2 n-1) f_{3}\right)\{\eta(Y) X-\eta(X) Y\} \eta(Z) \\
& \left.-\left(3 f_{2}+(2 n-1) f_{3}\right)\{g(Y, Z) \eta(X)-g(X, Z) \eta(Y)\} \xi\right] .
\end{aligned}
$$

Now, replacing $\mathrm{Z}$ by $\phi Z$ in the above equation, we obtain

$$
\begin{aligned}
& f_{1}\{g(Y, \phi Z) X-g(X, \phi Z) Y\}+f_{2}\{-g(X, Z) \phi Y+g(Y, Z) \phi X \\
& -2 g(X, \phi Y) Z+\eta(X) \eta(Z) \phi Y-\eta(Y) \eta(Z) \phi X+2 \eta(Z) g(X, \phi Y) \xi\} \\
& +f_{3}\{g(X, \phi Z) \eta(Y)-g(Y, \phi Z) \eta(X)\} \xi \\
& =\frac{1}{2(n-1)}\left[2\left(2 n f_{1}+3 f_{2}-f_{3}\right)\{g(Y, \phi Z) X-g(X, \phi Z) Y\}\right. \\
& \left.-\left(3 f_{2}+(2 n-1) f_{3}\right)\{g(Y, \phi Z) \eta(X)-g(X, \phi Z) \eta(Y)\} \xi\right],
\end{aligned}
$$

which by putting $X=\xi$ takes the form

$$
-2\left(f_{1}-f_{3}\right) g(Y, \phi Z) \xi=0 .
$$

Then either

$$
f_{1}=f_{3}
$$

or

$$
g(Y, \phi Z)=0 .
$$

Suppose $g(Y, \phi Z)=0$. Replacing $Z$ by $\phi Z$ in the equation (4.6) yields

$$
g\left(Y, \phi^{2} Z\right)=0,
$$


which implies

$$
Z=\eta(Z) \xi
$$

This shows that $Z$ is collinear with $\xi$.

Again replacing $\mathrm{X}$ by $\phi X$ in the equation 4.3 , we get

$$
\begin{aligned}
& f_{1}\{g(Y, Z) \phi X-g(\phi X, Z) Y\}+f_{2}\{g(X, Z) \phi Y-\eta(X) \eta(Z) \phi Y \\
& +g(Y, \phi Z) X-g(Y, \phi Z) \eta(X) \xi+2 g(X, Y) \phi Z-2 \eta(X) \eta(Y) \phi Z\} \\
& +f_{3}\{-\eta(Y) \eta(Z) \phi X+g(\phi X, Z) \eta(Y) \xi\} \\
& =\frac{1}{2(n-1)}\left[2\left(2 n f_{1}+3 f_{2}-f_{3}\right)\{g(Y, Z) \phi X-g(\phi X, Z) Y\}\right. \\
& \left.-\left(3 f_{2}+(2 n-1) f_{3}\right) \eta(Y) \eta(Z) \phi X+\left(3 f_{2}+(2 n-1) f_{3}\right) g(\phi X, Z) \eta(Y) \xi\right] .
\end{aligned}
$$

Now putting $Y=\xi$ in the above equation, we obtain

$$
\left[(2 n+1) f_{1}+3 f_{2}-3 f_{3}\right](\eta(Z) \phi X-g(\phi X, Z) \xi)=0 .
$$

Since $\eta(Z) \phi X-g(\phi X, Z) \xi \neq 0$ in general, we obtain

$$
(2 n+1) f_{1}+3 f_{2}-3 f_{3}=0 .
$$

From the equations 4.5 and 4.8, we have

$$
f_{1}=\frac{3 f_{2}}{2(1-n)} .
$$

Thus, in view of the equations 4.5 and 4.9), we have

$$
f_{1}=\frac{3 f_{2}}{2(1-n)}=f_{3}
$$

Conversely, suppose $f_{1}=\frac{3 f_{2}}{2(1-n)}=f_{3}$ satisfies a generalized Sasakian-space-form, then we have

$$
\begin{gathered}
S(X, Y)=0, \\
Q X=0 .
\end{gathered}
$$

Also, in view of the equation (2.16), we have

$$
{ }^{\prime} W^{*}(X, Y, Z, U)={ }^{\prime} R(X, Y, Z, U),
$$

where ${ }^{\prime} W^{*}(X, Y, Z, U)=g\left(W^{*}(X, Y) Z, U\right)$ and ' $R(X, Y, Z, U)=g(R(X, Y) Z, U)$. Putting $Y=Z=e_{i}$ in the equation (4.13) and taking summation over $i, 1 \leq i \leq$ $2 n+1$, we get

$$
\sum_{i=1}^{2 n+1}{ }^{\prime} W^{*}\left(X, e_{i}, e_{i}, U\right)=\sum_{i=1}^{2 n+1}{ }^{\prime} R\left(X, e_{i}, e_{i}, U\right)=S(X, U) .
$$


In view of the equations (4.13) and (2.6), we have

$$
\begin{aligned}
{ }^{\prime} W^{*}(X, Y, Z, U) & =f_{1}\{g(Y, Z) g(X, U)-g(X, Z) g(Y, U)\} \\
& +f_{2}\{g(X, \phi Z) g(\phi Y, U)-g(Y, \phi Z) g(\phi X, U) \\
& +2 g(X, \phi Y) g(\phi Z, U)\}+f_{3}\{\eta(X) \eta(Z) g(Y, U) \\
& -\eta(Y) \eta(Z) g(X, U)+g(X, Z) \eta(Y) \eta(U)-g(Y, Z) \eta(X) \eta(U)\} .
\end{aligned}
$$

Now, putting $Y=Z=e_{i}$ in the above equation and taking summation over $i$, $1 \leq i \leq 2 n+1$, we get

$$
\begin{aligned}
\sum_{i=1}^{2 n+1}{ }^{\prime} W^{*}\left(X, e_{i}, e_{i}, U\right) & =2 n f_{1} g(X, U)+3 f_{2} g(\phi X, \phi U) \\
& -f_{3}\{(2 n-1) \eta(X) \eta(U)+g(X, U)\} .
\end{aligned}
$$

In view of the equations 4.16), 4.14 and 4.11, we have

$$
2 n f_{1} g(X, U)+3 f_{2} g(\phi X, \phi U)-f_{3}\{(2 n-1) \eta(X) \eta(U)+g(X, U)\}=0 .
$$

Putting $X=W=e_{i}$ in the above equation and taking summation over $i, 1 \leq i \leq$ $2 n+1$, we get $f_{1}=0$. Then in view of the equation (4.10), $f_{2}=f_{3}=0$. Therefore, we obtain from the equation (2.6)

$$
R(X, Y) Z=0 .
$$

Hence in view of the equations 4.18, 4.11) and 4.12, we have $W^{*}(X, Y) Z=0$. This completes the proof.

\section{5. $\quad \xi$-m-Projectively Flat Generalized Sasakian-Space-Forms}

Definition 5.1. A $(2 n+1)$-dimensional $(n>1)$ generalized Sasakian-space-form is said to be $\xi$-m-projectively flat [14, if $W^{*}(X, Y) \xi=0$ for all $X, Y \in T M$.

Theorem 5.1. $A(2 n+1)$-dimensional $(n>1)$ generalized Sasakian-space-form is $\xi$-m-projectively flat if and only if it is an $\eta$-Einstein manifold.

Proof: Let us consider a generalized Sasakian-space-form is $\xi$-m-projectively flat, i.e. $W^{*}(X, Y) \xi=0$. Then in view if equation (2.16), we have

$$
R(X, Y) \xi=\frac{1}{2(n-1)}[S(Y, \xi) X-S(X, \xi) Y+g(Y, \xi) Q X-g(X, \xi) Q Y] .
$$

By virtue of the equations $2.2,2.10$ and 2.13 the above equation reduces to

$$
\eta(Y) Q X-\eta(X) Q Y=-2\left(f_{1}-f_{3}\right)[\eta(Y) X-\eta(X) Y],
$$


which by putting $Y=\xi$ gives

$$
Q X=2\left(f_{1}-f_{3}\right)[-X+(n+1) \eta(X) \xi] .
$$

Now, taking the inner product of the above equation with $\mathrm{U}$, we get

$$
S(X, U)=2\left(f_{1}-f_{3}\right)[-g(X, U)+(n+1) \eta(X) \eta(U)],
$$

which shows that generalized Sasakian-space-form is an $\eta$-Einstein manifold. Conversely, suppose the equation (5.4) is satisfied. Then by virtue of the equations (5.3) and (5.1), we have $W^{*}(X, Y) \xi=0$. This completes the proof.

\section{6. m-Projectively Recurrent Generalized Sasakian-Space-Forms}

Definition 6.1. A non-flat Riemannian manifold $M^{2 n+1}$ is said to be m-projectively recurrent if its m-projective curvature tensor $W^{*}$ satisfies the condition

$$
\nabla W^{*}=A \otimes W^{*},
$$

where $\mathrm{A}$ is a non-zero 1-form.

Theorem 6.1. If a $(2 n+1)$-dimensional $(n>1)$ generalized Sasakian-space-form is m-projectively recurrent, then either $f_{1}=f_{3}$ or it is an Einstein manifold.

Proof: We define a function $f^{2}=g\left(W^{*}, W^{*}\right)$ on $M^{2 n+1}$, where the metric $\mathrm{g}$ is extended to the inner product between the tensor fields. Then we have

$$
f(Y f)=f^{2} A(Y) .
$$

This can be written as

$$
Y f=f(A(Y)),(f \neq 0) .
$$

From the above equation, we have

$$
X(Y f)-Y(X f)=\{X A(Y)-Y A(X)-A([X, Y])\} f .
$$

Since the left hand side of the above equation is identically zero and $f \neq 0$ on $M^{2 n+1}$. Then

$$
d A(X, Y)=0
$$

i.e. 1-form $\mathrm{A}$ is closed.

Now from

$$
\left(\nabla_{Y} W^{*}\right)(Z, U) V=A(Y) W^{*}(Z, U) V
$$

we have

$$
\left(\nabla_{X} \nabla_{Y} W^{*}\right)(Z, U) V=\{X A(Y)+A(X) A(Y)\} W^{*}(Z, U) V .
$$


In view of the equations $(6.3)$ and $(6.4)$, we have

$$
\begin{aligned}
\left(R(X, Y) . W^{*}\right)(Z, U) V & =[2 d A(X, Y)] W^{*}(Z, U) V \\
& =0
\end{aligned}
$$

Thus in view of Theorem (3.1), we have either $f_{1}=f_{3}$ or $M^{2 n+1}$ is an Einstein manifold.

\section{Generalized Sasakian-Space-Forms Satisfying $W^{*} . S=0$.}

Theorem 7.1. A $(2 n+1)$-dimensional $(n>1)$ generalized Sasakian-space-form satisfying $W^{*} . S=0$ is an $\eta$-Einstein manifold.

Proof: Let us consider a generalized Sasakian-space-form $M^{2 n+1}$ satisfying $W^{*}(\xi, X) \cdot S=$ 0 . In this case we can write

$$
S\left(W^{*}(\xi, X) Y, Z\right)+S\left(Y, W^{*}(\xi, X) Z\right)=0 .
$$

In view of the equation 2.19 the above equation reduces to

$$
\begin{aligned}
& \left(f_{1}-f_{3}\right)\left[2 n\left(f_{1}-f_{3}\right)\{g(X, Y) \eta(Z)+g(X, Z) \eta(Y)\}\right. \\
& -\{\eta(Y) S(X, Z)+\eta(Z) S(X, Y)\}]+\frac{1}{2}\left[2 n\left(f_{1}-f_{3}\right)\{S(X, Y) \eta(Z)\right. \\
& +S(X, Z) \eta(Y)\}-\{\eta(Y) S(Q X, Z)+\eta(Z) S(Q X, Y)\}]=0 .
\end{aligned}
$$

Now, putting $Z=\xi$ in the above equation, we get

$$
S(Q X, Y)=2\left(f_{1}-f_{3}\right)\left[(n-1) S(X, Y)+2 n\left(f_{1}-f_{3}\right) g(X, Y)\right] .
$$

By virtue of the equation 2.7 the above equation takes the form

$$
S(X, Y)=\frac{2 n\left(f_{1}-f_{3}\right)}{K}\left[2\left(f_{1}-f_{3}\right) g(X, Y)+\left(3 f_{2}+(2 n-1) f_{3}\right) \eta(X) \eta(Y)\right],
$$

where $K=2 n f_{1}+3 f_{2}+(2 n-3) f_{3}$, which shows that $M^{2 n+1}$ is an $\eta$-Einstein manifold. This completes the proof.

\section{Generalized Sasakian-Space-Forms Satisfying $W^{*} \cdot R=0$.}

Theorem 8.1. A $(2 n+1)$-dimensional $(n>1)$ generalized Sasakian-space-form satisfying $W^{*} . R=0$ is an $\eta$-Einstein manifold. 
Proof: Suppose $M^{2 n+1}$ satisfying $\left(W^{*}(\xi, X) \cdot R\right)(Y, Z) U=0$, then it can be written as

$$
\begin{aligned}
& W^{*}(\xi, X) R(Y, Z) U-R\left(W^{*}(\xi, X) Y, Z\right) U-R\left(Y, W^{*}(\xi, X) Z\right) U \\
& -R(Y, Z) W^{*}(\xi, X) U=0,
\end{aligned}
$$

which on using the equation 2.19 takes the form

$$
\begin{aligned}
& \frac{\left(f_{1}-f_{3}\right)}{(n-1)}[-g(X, R(Y, Z) U) \xi+\eta(R(Y, Z) U) X+g(X, Y) R(\xi, Z) U \\
& -\eta(Y) R(X, Z) U+g(X, Z) R(Y, \xi) U-\eta(Z) R(Y, X) U \\
& +g(X, U) R(Y, Z) \xi-\eta(U) R(Y, Z) X]-\frac{1}{2(n-1)}[S(X, R(Y, Z) U) \xi \\
& -\eta(R(Y, Z) U) Q X-S(X, Y) R(\xi, Z) U+\eta(Y) R(Q X, Z) U \\
& -S(X, Z) R(Y, \xi) U+\eta(Z) R(Y, Q X) U-S(X, U) R(Y, Z) \xi \\
& +\eta(U) R(Y, Z) Q X]=0 .
\end{aligned}
$$

Taking the inner product of the above equation with $\xi$, we get

$$
\begin{aligned}
& \frac{\left(f_{1}-f_{3}\right)}{(n-1)}[-g(X, R(Y, Z) U)+\eta(R(Y, Z) U) \eta(X)+g(X, Y) \eta(R(\xi, Z) U) \\
& -\eta(Y) \eta(R(X, Z) U)+g(X, Z) \eta(R(Y, \xi) U)-\eta(Z) \eta(R(Y, X) U) \\
& +g(X, U) \eta(R(Y, Z) \xi)-\eta(U) \eta(R(Y, Z) X)]-\frac{1}{2(n-1)}[S(X, R(Y, Z) U) \\
& -\eta(R(Y, Z) U) \eta(Q X)-S(X, Y) \eta(R(\xi, Z) U)+\eta(Y) \eta(R(Q X, Z) U) \\
& -S(X, Z) \eta(R(Y, \xi) U)+\eta(Z) \eta(R(Y, Q X) U)-S(X, U) \eta(R(Y, Z) \xi) \\
& +\eta(U) \eta(R(Y, Z) Q X)]=0 .
\end{aligned}
$$

Now using the equations $(2.6), 2.11)$ and 2.12 in the above equation, we get

$$
\begin{aligned}
& \left(f_{1}-f_{3}\right)\left[-f_{1}\{g(Z, U) g(X, Y)-g(Y, U) g(X, Z)\}\right. \\
& -f_{2}\{g(Y, \phi U) g(\phi Z, X)-g(Z, \phi U) g(\phi Y, X)+2 g(Y, \phi Z) g(\phi U, X)\} \\
& -f_{3}\{\eta(Y) \eta(U) g(X, Z)-\eta(Z) \eta(U) g(X, Y)+g(Y, U) \eta(Z) \eta(X) \\
& \left.-g(Z, U) \eta(Y) \eta(X)\}+\left(f_{1}-f_{3}\right)\{g(Z, U) g(X, Y)-g(Y, U) g(X, Z)\}\right] \\
& -\frac{1}{2}\left[f_{1}\{g(Z, U) S(X, Y)-g(Y, U) S(X, Z)\}+f_{2}\{g(Y, \phi U) S(\phi Z, X)\right. \\
& -g(Z, \phi U) S(\phi Y, X)+2 g(Y, \phi Z) S(\phi U, X)\}+f_{3}\{\eta(Y) \eta(U) S(X, Z) \\
& -\eta(Z) \eta(U) S(X, Y)+2 n\left(f_{1}-f_{3}\right) \eta(X) \eta(Z) g(Y, U) \\
& \left.-2 n\left(f_{1}-f_{3}\right) \eta(X) \eta(Y) g(Z, U)\right\}-\left(f_{1}-f_{3}\right)\{g(Z, U) S(X, Y) \\
& -g(Y, U) S(X, Z)\}]=0 .
\end{aligned}
$$

Putting $Z=U=e_{i}$ in the above equation and summing over i, $1 \leq i \leq 2 n+1$, we get

$$
S(X, Y)=2\left(f_{1}-f_{3}\right)[-g(X, Y)+(n+1) \eta(X) \eta(Y)]
$$


which shows that $M^{2 n+1}$ is an $\eta$-Einstein manifold. This completes the proof.

\section{REFEREN CES}

1. P. Alegre, D. E. Blair and A. CARriazo: Generalized Sasakian-space-forms, Israel J. Math., 141(2004),151-183.

2. P. Alegre and A. CARriazo:Structures on generalized Sasakian-space-forms, Differential Geom. and its Application, 26(2008), 656-666.doi 10.1016/J.difgeo.

3. P. Alegre, A. Carriazo, Y. H. Kim and D. W. Yoon: B. Y. Chen's innequality for submanifolds of generalized space forms, Indian J. Pure Appl. Math., 38(2007), 185-201.

4. R. Al-Ghefari, F. R. Al-Solamy and M. H. Shahid: CR-Submanifolds of generalized Sasakian-space-forms, JP J. Geom.Topol., 6(2006), 151-166.

5. R. Al-Ghefari, F. R. Al-Solamy and M. H. Shahid: Contact CR-warped product submanifolds in generalized Sasakian-space-forms, Balkan J. Geom., Appl., 11(2)(2006), 1-10.

6. D. E. BLAIR: Contact manifolds in a Riemannian geometry, Lecture notes in Math., 509, Springer Verlag, Verlin, 1976.

7. U. C. DEand AVIJIT SARKAR: On the projective curvature tensor of generalized Sasakian-space-forms, Quaestiones Mathematicae, 33(2010), 245-252.

8. U. C. DE, R. N. Singh and S. K. PANDEY: On the conharmonic curvature tensor of generalized Sasakian-space-forms,ISRN Geometry, 14 Pages, doi:10.5402/2012/876276, 2012.

9. U. K. KIM: Conformally flat generalized Sasakian-space-forms and locally symmetric generalized Sasakian-space-forms, Note di Mathematica,26(2006), 55-67.

10. R. H. Олна: M-Projectively flat Sasakian manifolds, Indian J. Pure Appl. Math.,17(4)(1986), 481-484.

11. C. ÖzGüR: On $\phi$ - conformally flat Lorentzian para Sasakian manifolds, Radovi Mathematicki,12(1)(2003), 99-106.

12. G. P. Pokhariyal and R. S. Mishra: Curvature tensor and their relativistic significance II, Yokohama Mathematical Journal,19(1971), 97-103.

13. E. A. PolkinA: Curvature identities for almost contact metric manifolds, Russian Mathematics (IZ VUZ), 51(7)(2007), 54-57.

14. G. Zhen, J. L. Cabrerizo, L. M. Fernández and M. Fernández: On $\xi$ conformally flat contact metric manifolds, Indian J.Pure Appl. Math.,28(1997), 725-734.

Shravan K. Pandey

Department of Mathematical Sciences

A.P.S.University, Rewa-486003 (M.P.), India

shravan.math@gmail.com 
R.N.Singh

Department of Mathematical Sciences

A.P.S.University, Rewa-486003 (M.P.), India

rnsinghmp@rediffmail.com 